\title{
Abnormalities in the brain-immune system in psychotic disorders: from pathogenesis to prevention
}

\author{
Kenji Hashimoto ${ }^{1}$
}

Published online: 7 June 2017

(C) Springer-Verlag GmbH Germany 2017

\section{Editorial:}

Multiple lines of evidence suggest that abnormalities in the brain-immune system play a key role in the pathogenesis of psychotic disorders [1]. Accumulating data suggest that changes in the function of the immune system, particularly an increase in inflammation, can negatively impact virtually every aspect of brain function, including neurogenesis, synaptic remodeling, neuronal excitability, metabolic support, and even the response to medication [1]. The best-documented and most reproducible findings are increases in peripheral blood levels of interleukin-6 (IL6) and C-reactive protein (CRP) in patients with psychotic disorders. Although the precise mechanisms underlying the inflammatory response in these patients are unknown, it has been reported that there is increased activation of fundamental inflammatory signaling molecules and pathways [1]. Therefore, appropriate compounds which promote or regulate certain elements of immune system function may prevent the onset of psychotic disorders.

Blood IL-6 and high-sensitivity CRP (hsCRP) levels have been observed to be significantly higher in schizophrenia patients $(n=151)$ than in healthy controls $(n=194)$ [2]. Interestingly, the levels of both IL-6 and hsCRP in that study were associated with the insidious onset of the psychosis, the duration of the illness, and a chronic schizophrenia course with deterioration. These results suggest that elevated blood levels of IL-6 may serve as an inflammatory biomarker of deterioration in schizophrenia [2].

Kenji Hashimoto

hashimoto@faculty.chiba-u.jp

1 Division of Clinical Neuroscience, Chiba University Center for Forensic Mental Health, 1-8-1 Inohana, Chiba 260-8670, Japan
In this issue, Braun et al. [3] report a study in which they took four independent blood samples from patients with psychotic disorders, including schizophrenia $(n=140)$, schizoaffective disorder $(n=43)$, bipolar disorder $(n=34)$, and depression $(n=52)$ with psychotic symptoms, and measured levels of immunoglobulin M (IgM) and the cytokines IL- 6 , tumor necrosis factor- $\alpha$, neopterin, transforming growth factor- $\beta 1$, and soluble cluster of differentiation 14 (sCD14). In a subgroup of $20-30 \%$ of the patients, they noted unusual behavior of the inflammatory response system, as quantified through IgM levels, that appeared to be associated with the pathogenesis of schizophrenia $(p<0.0001)$. They also report that the variation in concordance for schizophrenia of monozygotic twins could in part be "explained" by chronically elevated IgM levels (accounting for $24.54 \%$ of the observed phenotypic variance; $p=0.043$ ), suggesting that monozygotic twins who are concordant for schizophrenia may possess a less "robust" variant of the inflammatory response system. This paper suggests a way for the early identification of the patients with schizophrenia for whom the inflammatory response system may be a target for therapeutic intervention [3].

Cerebrospinal fluid (CSF) is a more appropriate sampling substrate than blood because it more closely reflects brain functions. In this issue, Johansson et al. [4] report measuring CSF biomarkers of microglia activation, including monocyte chemoattractant protein-1, chitinase 3-like protein 1 (YKL-40), and sCD14, in 17 pairs of twins, including those with schizophrenia or bipolar disorder and those who were not affected by these disorders. They found higher CSF levels of sCD14 in the subjects with schizophrenia or bipolar disorder compared to their unaffected co-twins. sCD14 is produced in the central nervous system by activated macrophages triggered by the immune system, 
and it has been suggested that it is in involved in microglial activation. Johansson et al.'s study provides support for a role of $\mathrm{sCD} 14$, and a possible role of microglial activation, in psychotic disorders [4].

Monocyte activation has consistently been reported in patients with schizophrenia. Peripheral monocytes share similarities with microglia in phagocytic activities. When activated, monocytes release pro-inflammatory cytokines, including interleukin-1 $\beta$ (IL-1 $\beta$ ), in response to stimuli in the brain under various pathological conditions [5]. In this issue, Uranova et al. [6] report the ultrastructure of monocytes and the monocyte production of IL- $1 \beta$ in drug-free young patients with schizophrenia $(n=9)$ and healthy young control subjects $(n=11)$. The area of the nucleolus, the volume density and areas of mitochondria and lysosomes, and the number of lysosomes were significantly increased in the patients as compared to the controls. Furthermore, the area of mitochondria correlated significantly with the volume density and area of lysosomes. In addition, the production of IL-1 $\beta$ by monocytes was significantly higher in the patients than in the controls. Interestingly, there were positive correlations between the production of IL-1 $\beta$ and the volume density of lysosomes $(r=0.78$, $p=0.013)$ and the area of lysosomes $(r=0.68, p=0.04)$ in the patients with schizophrenia. Even though the sample size of this study was small, these findings provide new evidence that monocytes are overactivated in schizophrenia and that the metabolic relationship between lysosomes, mitochondria, and vacuoles is disturbed [6]. Together, the results suggest it is likely that monocytes would be suitable biomarker targets for the diagnosis and prognosis of psychotic disorders.

Prenatal infection is involved in the etiology of neurodevelopmental disorders, including schizophrenia and autism. Accumulating data show that maternal immune activation in rodents has a considerable impact on brain development and behavioral abnormalities in their offspring [7]. The juvenile offspring of rodents exposed during pregnancy to polyinosinic-polycytidylic acid [poly(I:C)] mimic the behavioral abnormalities associated with the prodromal stage of schizophrenia, such as cognitive deficits [7]. When adult, these rodents can mimic both the behavioral abnormalities (such as cognitive deficits and prepulse inhibition) and the loss of parvalbumin-positive cells in the prefrontal cortex (PFC) observed in patients with schizophrenia [7]. It is well known that brain-derived neurotrophic factor (BDNF) and its receptor TrkB play a crucial role in the neurodevelopment and inflammatory responses. Han et al. [7] have reported that decreased BDNF-TrkB signaling in the PFC may play a role in the cognitive deficits observed in the offspring of poly(I:C)-treated mice. Conversely, supplementation with the naturally occurring TrkB agonist 7,8-dihydroxyflavone (7,8-DHF) during the juvenile and adolescent stages of these mice can prevent the onset of schizophrenia-like behavioral abnormalities and the loss of parvalbumin-positive cells in the PFC when adult [7]. These findings suggest that decreased BDNFTrkB signaling plays a role in the pathophysiology of neurodevelopmental disorders such as schizophrenia, and that supplementation with a TrkB agonist during the juvenile and adolescent stages may prevent the onset of psychotic disorders in adulthood.

It is also known that intrauterine nutritional deficiency may increase the risk of psychiatric disorders such as schizophrenia. In this issue, Han et al. [8] report that supplementation with 7,8-DHF administered to poly(I:C)-treated pregnant mice from pregnancy to weaning could prevent the onset of cognitive deficits and reduced BDNF-TrkB signaling in the PFC of their adult offspring. This study suggests that administering supplementation with a TrkB agonist to pregnant women with an ultra-high risk of psychosis may reduce the development of psychosis in their offspring [8].

In recent years, there have been increasing reports about the involvement of the gut-microbiota-brain axis in the pathogenesis of psychotic disorders. One key pathway through which this axis may affect brain function is modulation of the autonomic nervous system, including via activation of the vagus nerve. Another pathway is via microbial signaling molecules, including short-chain fatty acids produced by gut microorganisms [9]. Abnormality in the composition of the gut microbiota can also affect brain function through the immune system, indicating that the gut microbiota communicates with brain function activating immune signaling pathways, including through the release of cytokines (e.g., IL-6) and other inflammatory molecules [9]. In a recent study of susceptible mice whose gut microbiota had been altered by chronic social defeat stress, the blockade of IL-6 receptors in the periphery promoted rapid and sustained antidepressant actions by normalizing the composition of the gut microbiota [10]. Therefore, the blockade of IL- 6 receptors in the periphery shows promise as a novel therapeutic approach for patients with higher IL-6 blood levels since the humanized anti-IL-6 receptor antibody tocilizumab has been used in the treatment of patients with rheumatoid arthritis. Taken together, these findings suggest that it is likely that the gut-microbiota-brain axis plays a role in abnormalities in the brainimmune system in psychotic disorders.

In conclusion, abnormalities in the brain-immune system, including inflammation, play a key role in the pathogenesis of psychotic disorders. Early intervention involving appropriate nutrition with anti-inflammatory actions may prevent the onset of psychotic disorders in adolescence and adulthood. 
Acknowledgements This study was supported by a Grant from the Strategic Research Program for Brain Sciences, Agency for Medical Research and Development (AMED), Japan.

\section{Compliance with ethical standards}

Conflict of interest The author declares that they have no competing interests.

\section{References}

1. Miller AD, Haroon E, Felger JC (2017) Therapeutic implications of brain-immune interactions: treatment in translation. Neuropsychopharmacology 44:334-359

2. Frydecka D, Misiak B, Pawlak-Adamska E, Karabon L, Tomkiewicz A, Sedlaczek P, Kiejna A, Beszłej JA (2016) Interleukin-6: the missing element of the neurocognitive deterioration in schizophrenia? The focus on genetic underpinnings, cognitive impairment and clinical manifestation. Eur Arch Psychiatry Clin Neurosci 265:449-459

3. Braun S, Bridler R, Müller N, Schwarz MJ, Seifritz E, Weisbrod M, Zgraggen A, Stassen HH (2017) Inflammatory processes and schizophrenia: two independent lines of evidence from a study of twins discordant and concordant for schizophrenic disorders. Eur Arch Psychiatry Clin Neurosci. doi:10.1007/s00406-017-0792-Z
4. Johansson V, Jakobsson J, Fortgang RG, Zetterberg H, Blennow K, Cannon TD, Hultman CM, Wetterberg L, Landén M (2017) Cerebrospinal fluid microglia and neurodegenerative markers in twins concordant and discordant for psychotic disorders. Eur Arch Psychiatry Clin Neurosci. doi:10.1007/s00406-016-0759-5

5. Takahashi Y, Yu Z, Sakai M, Tomita H (2016) Linking activation of microglia and peripheral monocytic cells to the pathophysiology of psychiatric disorders. Front Cell Neurosci 10:144

6. Uranova NA, Bonartsev PD, Androsova LV, Rakhmanova VI, Kaleda VG (2017) Impaired monocyte activation in schizophrenia: ultrastructural abnormalities and increased IL-1 $\beta$ production. Eur Arch Psychiatry Clin Neurosci. doi:10.1007/ s00406-017-0782-1

7. Han M, Zhang JC, Yao W, Yang C, Ishima T, Ren Q, Ma M, Dong C, Huang XF, Hashimoto K (2016) Intake of 7,8-dihydroxyflavone during juvenile and adolescent stages prevents onset of psychosis in adult offspring after maternal immune activation. Sci Rep 6:36087

8. Han M,Zhang JC, Huang XF, Hashimoto K(2017) Intake of 7,8-dihydroxyflavone from pregnancy to weaning prevents cognitive deficits in adult offspring after maternal immune activation. Eur Arch Psychiatry Clin Neurosci. doi:10.1007/s00406-017-0802-1

9. Ho P, Ross DA (2017) More than a gut feeling: the implications of the gut microbiota in psychiatry. Biol Psychiatry 81:e35-e37

10. Zhang JC, Yao W, Dong C, Yang C, Ren Q, Ma M, Hashimoto K (2017) Blockade of interleukin-6 receptor in the periphery promotes rapid and sustained antidepressant actions: a possible role of gut-microbiota-brain axis. Transl Psychiatry 7:e1138 